\title{
Psychopathologie der Erleuchtung
}

\author{
Psychiatrisch-linguistische Lektüren spiritueller \\ Erwachenserzählungen
}

\section{Normalität und die Ordnung des Außerordentlichen}

Psychiatrische Diagnosen basieren auf einer seltsamen, doppelten Binarität von gesund - krank und normal - krank (Finzen 2018). Der Ausdruck krank (mit den verwandten Ausdrücken psychisch krank, verrückt u.a.) hat zwei Gegensätze: normal und gesund. Damit werden gesund und normal teilsynonym, d.h. sie werden in bestimmten Kontexten in gleicher Weise verwendet. Das Gesunde ist damit zumindest teilweise gleichbedeutend mit dem Normalen. Daher argumentiert der Psychiater Asmus Finzen (2018), dass der Normalitätsbegriff immer auch in medizinischen und psychiatrischen Diagnosen enthalten sei. Diese Unterscheidung und die jeweiligen Grenzen, die durch sie gezogen werden, sind historisch bedingt, wie Foucault in seinem Werk Wahnsinn und Gesellschaft gezeigt hat (Foucault 2018). Die Grenzen des Normalen verlaufen aber nicht nur in der Zeit, sondern auch intra- und interkulturell innerhalb eines Zeitschnittes. Während intrakulturelle Normalitätsverschiebungen noch mit dem Als-Ob-Modus verstanden werden können (eine Person hat sich beispielsweise als Prinzessin verkleidet, weiß aber, dass sie keine ist), ist dies bei interkulturell verschiedenen Normalitäten nicht mehr ganz so einfach mit dem Als-Ob-Modus erklärbar. Im Falle von anderen Normalitäten unterschiedlicher Kulturen argumentieren einige Ethnolog^innen wie Viveiros de Castro (2016), dass man von verschiedenen Ontologien, also verschiedenen Wirklichkeiten ausgehen müsse. Was normal ist, variiert also in und zwischen den Kulturen. In der Karnevalszeit gilt eine andere Normalität, in anderen Kulturen gibt es andere außerordentliche Zeiten, die eine eigene Ordnung aufweisen. Wer im Karneval als Protagonist auftreten will, muss sich kostümieren, aber nur hier, kommt er außerhalb der Karnevalszeit so zur Arbeit oder geht etwa mit einem Zorrokostüm in eine Bank, wird ihm sein Irrtum saisonaler Normalität schmerzhaft bewusst gemacht werden. Sektionale Normalität: Der Schamane wird als ,vorwissenschaftlich' des Feldes der Rationalität verwiesen. Normalität, ob sektional oder saisonal, wird damit als Diskursprozess und Diskursfaktor deutlich, die sich beide mit Macht

Wolf-Andreas Liebert, Universität Koblenz-Landau, Campus Koblenz, Institut für Germanistik, liebert@uni-koblenz.de

Ә Open Access. ( 2021 Wolf-Andreas Liebert, publiziert von De Gruyter. (c) BY-NC-ND Dieses Werk ist lizenziert unter einer Creative Commons Namensnennung - Nicht-kommerziell - Keine Bearbeitung 4.0 International Lizenz. https://doi.org/10.1515/9783110688696-027 
gegen ein ungeregeltes Überschreiten der Grenzen stemmen. Immer wenn diese Grenzen nicht eingehalten werden, droht das Nichtnormale zu einem Kranken zu werden, das durch entsprechende Behandlung verändert werden muss.

\section{Ist Religion der Wahnsinn?}

Doch wie verhält sich dies mit Religionen? Religion gehört zweifellos zur Normalität - oder nicht? In meiner Beschäftigung mit Erwachenserzählungen (Liebert i. E.) ist mir aufgefallen, dass es in sprachlicher Hinsicht Ähnlichkeiten mit Texten psychisch Kranker gibt. Manchmal musste nur der kulturelle Kontext verändert werden, um statt eines religiösen Textes einen „Fall“ zu haben, ebenso konnte ein „Fall“ auch als - im psychiatrischen Sinne - „nicht abweichend“ gedeutet werden, wenn angenommen wurde, der oder die Sprechende sei ein gläubiger Mensch.

Ist Glaube also ein Kontext, der für saisonale oder sektionale Normalität entscheidend ist? Gibt es ein Verhältnis von Texten psychisch kranker und religiöser Menschen? Und was können wir daraus für unser Verständnis von Normalität und ihrer sprachlich-kulturellen Konstitution lernen? Die Behandlung dieser Fragen wird durch die Kenntnis einer weiteren Frage bedingt, nämlich wie Religion und Religiosität in der Psychiatrie und Psychotherapie selbst aufgefasst und bewertet werden.

Daran schließt sich eine weitere Frage an, nämlich wie Religion und Religiosität in der Psychiatrie und Psychotherapie selbst aufgefasst und bewertet werden. Müssten Moses oder Abraham heute als Psychotiker in die psychiatrische Klinik, weil sie göttliche Stimmen gehört haben und sich letzterer dieser Stimme auch dann noch unterworfen hat, als die Stimme ihn zum Opfermord seines Sohnes aufforderte? Ist Religion also per se Wahnsinn? Würde ein:e Psychiater:in diese Frage zu Gesicht bekommen, würde sie diese empört zurückweisen und zu Recht auf die lange Tradition der Beschäftigung der Psychiatrie mit dem Phänomenfeld Religiosität verweisen. Jedoch dürften sich in naturwissenschaftlichen Disziplinen, als die sich die Psychiatrie zumeist versteht, durchaus solche skeptischen Positionen zeigen. Für Sigmund Freud war Religion nur eine Illusion, eine Kindheitsphantasie, für Karl Marx ein traumerzeugendes Sedativ, um Machtverhältnisse zu zementieren. Allein durch die Möglichkeit dieser Frage manifestiert sich eine historische Verschiebung, was normal ist und was nicht. Der Glaube an ein wie auch immer geartetes Göttliches, das jenseits dieser Welt auf diese Welt machtvoll einwirkt, war bis ins 19. Jahrhundert in den meisten Kulturen Europas das „Normale“. Das atheistisch-materialistische Paradigma wird erst im Verlauf der Aufklärung und mit der Erzeugung von Herrschaftswissen mächtig. 
Mehr und mehr musste man sich zu einer transzendenten oder einer nichttranszendenten Haltung positionieren (vgl. Liebert 2017, 2018): Glaubst du oder glaubst du nicht? Die Gretchenfrage wird im Faust des 19. Jahrhunderts gestellt. Die Positionierung: transzendent oder non-transzendent ist spätestens seit dieser Zeit zu einem agonalen Zentrum ${ }^{1}$ geworden. Kurze Zeit schien es so, als würde die transzendente Positionierung im 20. Jahrhundert einer Säkularisierung zum Opfer fallen und sich auflösen. Seitdem aber säkulare Ordnungen wieder zerbrochen und neue Fundamentalismen entstanden sind, erscheint die transzendente Positionierung in neuen Formen wieder machtvoll in der globalisierten Welt. ${ }^{2}$ Daher muss sich auch die Psychiatrie - zähneknirschend soweit sie atheistisch positioniert ist - mit dem Thema Religion verstärkt beschäftigen und die Grenze zwischen ,normalpsychologischer‘ Religiosität und krankhaften Fällen wie religiösem Wahn, Psychosen und anderen Erkrankungen ziehen: Wie erkenne ich, ob jemand einfach religiös ist oder als krank diagnostiziert werden muss, ob hier eine ,Störung، vorliegt? Im Pragmatismuskonzept von William James (1997) ist es schön einfach, da wird die Validität religiöser Erfahrung an den Ergebnissen gemessen. Die einfachen Fälle sind die Religionsgründer, die mit genügend historischem Abstand als erfolgreich gelten können - ihnen kann eine authentische Religiosität zugesprochen werden. Doch was ist mit den vielen anderen? Und was ist mit den Gegenwärtigen? Auf Jesus und eine an ihn anschließende machtvolle Geschichte des Christentums hätten seine Zeitgenossen wohl nicht gesetzt. Woher weiß ich also als behandelnder Arzt, ob ich eine leidende Kreatur vor mir habe, die Stimmen hört, oder den Gründer einer neuen Religion, der sich dereinst in den Geschichtsbüchern verewigen wird, falls ich ihn nicht falsch diagnostiziere? Michael Schödlbauer spitzt dieses Dilemma am Beispiel eines Weltuntergangspropheten in der Fußgängerzone zu, an dem sich für ihn zeigt ,auf welch schwankendem Boden hier nicht nur der fragliche Prophet auf seiner Kiste, sondern auch der Psychiater mit seinem klinischen Urteil steht“" (Schödlbauer 2016: 262).

\section{Die Gretchenfrage stellt sich in der Psychiatrie anders als in der Religionslinguistik}

Das Themenfeld Religion, Spiritualität oder veränderte Bewusstseinszustände wird in der Psychiatrie unter dem Stichwort Religionspsychopathologie schon

1 Zur Agonalität vgl. Felder (2006).

2 Zur „postsäkularen Gesellschaft“ vgl. Habermas (2012: insb. 308-327). 
lange diskutiert (vgl. Schödlbauer 2016: 263-302). Seit den Nullerjahren gibt es eine zunehmende Zahl von Publikationen, in denen auch die Forderung erhoben wird, Religion und Spiritualität als Konzept in die Psychotherapie, etwa als Copingstrategie oder Resilienzstärkung, zu integrieren (vgl. Bucher 2007; Grom 2007; Mönter 2007; Mundhenk 2010; Vaitl 2012; Reiser 2019). Diese Debatte ist ausgesprochen spannend. Doch - wie denken Psychiater*innen eigentlich? Während es noch Anfang des 20. Jahrhunderts verschiedene Schulen der Psychiatrie gab, hat der Druck, zu einheitlichen (d.h. standardisierten) Diagnosen und zu einer Vergleichbarkeit von Forschungsergebnissen zu kommen, in den letzten Dekaden zwei schon länger bestehende internationale Klassifikationssysteme zu mächtigen Akteuren heranwachsen lassen. Es handelt sich um das Werk International Statistical Classification of Diseases and Related Health Problems, kurz ICD, und das Diagnostic and Statistical Manual of Mental Disorders, kurz DSM, die beide auch in jeweils aktueller Auflage auf Deutsch im Hogrefe-Verlag erscheinen und je nach Auflage mit bestimmten Ziffern versehen werden (APA 2018; Dilling, Mombour \& Schmidt 2015). Religion spielt dabei eine untergeordnete Rolle, auch wenn im DSM deutlich mehr Dynamik erkennbar ist, was sich am Beispiel der Geschichte der Kategorie „Religiöses oder Spirituelles Problem“ zeigt (Lukoff 2017). Für die folgende Argumentation entscheidend ist der Objektivitätsanspruch, der etwa vom Hogrefe-Verlag für beide Werke reklamiert wird: „Beide Klassifikationen stellen eindeutige Störungskriterien für eine objektive Diagnostik bereit. “3 Beide Systeme stellen so eine internationale Verständigungsgrundlage dar, die aber von kulturellen Normalitätsvorstellungen durchzogen ist. So zeigt sich die kulturell konstruierte Antonymie von normal und gesund auch in diesen beiden Werken. Beispielsweise wurde in früheren Versionen Homosexualität noch als „Störung“ aufgeführt. Transsexualität ist derzeit noch eine „Störung“, soll aber in der nächsten Auflage des ICD als „Störung“ entfernt werden. In der aktuellen Fassung ist „Computerspielabhängigkeit“ als neue „Störung“ aufgenommen worden, die bis vor kurzem noch keine „Störung“ war. Auch wurde der Depressionsbegriff erweitert, der nun auch leichtere Formen wie emotionale Verstimmungen einschließt. Ist nun der statistische Anstieg von Depressionen auf die Invasion dieser Klassifikationen in bisher als normal geltende Bereiche zurückzuführen oder haben sie ,tatsächlich‘ zugenommen? Beide Diagnosesysteme, das ICD der WHO wie das DSM der American Psychiatric Association, basieren auf einem deskriptiven Symptommodell, mit dem eine ,Störung‘ in Bezug auf die jeweiligen Normalitäten

3 https://www.hogrefe.de/themen/klinik/artikeldetailansicht/DSM\%20und\%20ICD-313, letzter Zugriff: 14. 9. 2020. 
diagnostiziert, indem sie klassifiziert wird. Sie legt aber aus dem Störungsfeld heraus zuallererst fest, was normal ist und was Störung. Es könnte nun vorgebracht werden, diese Klassifikationssysteme passten sich ja offensichtlich der Entwicklung der Gesellschaft an, so seien etwa für den „Code F 44.3 Trance- und Besessenheitszustände“ religiöse Kontexte exkludiert worden. Diesem Argument könnte aber vorgehalten werden, es basiere auf einem naturalistischen Gesellschaftsbegriff, als sei immer schon klar, wie die Gesellschaft gerade aussehe und wie sie sich entwickle, als könne auf ein Klassifikationssystem von Gesellschaft zurückgegriffen werden. Für Gesellschaft und ihre Entwicklung gibt es aber kein solches Klassifikationssystem. Zudem würde eine solche Argumentation verkennen, dass der Psychiatrie keine Beobachterposition außerhalb der Gesellschaft zukommt, sondern dass die Psychiatrie als wichtiger Machtfaktor in der Gesellschaft wirkt.

Bevor jedoch überhaupt eine Situation zustande kommt, in der ein Psychiater mithilfe dieser Klassifikationssprachen Patienten diagnostiziert, geht der psychiatrischen Diagnose zunächst in der Regel ein leidender Mensch und eine Alltagsdiagnose psychiatrischer Laien, z.B. der Nachbarn, voraus, die im Wesentlichen in einer hermeneutischen Störung der Umwelt gründet: Die entsprechende Person leidet und verletzt möglicherweise Normen der sozialen Angepasstheit und ist in verschiedenen Verhaltens- und Kommunikationsdimensionen für ihre Mitmenschen unverständlich.

Wenn Menschen ,fruchtlos' sind, also nicht das realisieren, was in der jeweiligen Kultur als Erfolg gilt, dann müssen sich diejenigen, die von der Person eine Veränderung fordern, nicht besonders rechtfertigen. Der Rechtfertigungsdruck ist für diese psychiatrischen Störungsmodelle jedoch groß, wenn es sich dabei um Personen handelt, die zwar Merkmale psychischer Krankheiten zeigen, nach einer bestimmten Zeit jedoch in der Gesellschaft positiv herausgehoben und als besonders wertvoll anerkannt werden. Dazu zählen v.a. Innovatoren in den Kulturbereichen Technik, Philosophie, Literatur, Kunst, Musik und Religion. Das Feld der Religion ist hier besonders interessant, da es - wie eben bereits angedeutet selbst dem Diskurs über den Wahnsinn unterworfen ist (vgl. Freud 1927; Marx 1956; Foucault 2018; Schödlbauer 2016). Insofern hält der Diskurs auch eine Position bereit, die besonders gern von Atheisten vertreten wird, wonach allein das Bekenntnis zu einer Religion nicht nur ein Symptom anzeigt, sondern zugleich auch die Diagnose einer psychischen Störung bedeutet: „Der Begriff ,religiöser Wahn“ gilt unter Atheisten als Pleonasmus“ (Schödlbauer 2016: 262). Um diese und andere Selbstkategorisierungen und -beschreibungen ernst $\mathrm{zu}$ nehmen und keine verdeckten Vorab-Diagnosen zu erstellen, versucht die Religionslinguistik nicht nur transzendente, sondern auch nicht-transzendente Haltungen als Positionierungen zu verstehen (vgl. Liebert 2017, 2018). Die im 19. Jahrhundert aufgeworfene Gretchenfrage ist hier also keine moralische, sondern eine sachliche, die auch an 
den Psychiater gerichtet ist, selbst wenn dieser einen atheistischen Glauben haben sollte: „Nun sag', wie hast du's nicht mit der Religion?“ So ergeben sich aus dem Bisherigen zwei Hinsichten, die Religionslinguistik und psychiatrische Praxis vermitteln: Wie fassen zeitgenössische Berichte außergewöhnliche Erfahrungen im religiösen Bereich sprachlich und wie interpretieren Psychiaterinnen dies vor dem Hintergrund psychiatrisch-diagnostischer Deskriptoren?

\section{Psychiatrie und Religionslinguistik im Dialogischen Experteninterview}

Das Feld von Erwachenserzählungen ist in der Spätmoderne recht vielfältig. Diese Textsorte kann weit in die Vergangenheit zurückverfolgt werden und wurde vielfältig erforscht, auch in der Linguistik (vgl. Lasch 2005). Mein religionslinguistisches Interesse gilt in erster Linie der spätmodernen informellen Religiosität, die in der Religionswissenschaft und Religionssoziologie mit Begriffen wie Esoterik, Spiritualität oder Selbstermächtigung beschrieben wird (vgl. Gebhardt, Engelbrecht \& Bochinger 2005; Bochinger, Engelbrecht \& Gebhardt 2009; Knoblauch 2009). Die Erwachenserzählungen in diesem Feld können grob eingeteilt werden in prominente Literatur, die eine sehr große Reichweite besitzt, und graue Literatur, die in kleinen Auflagen oder im Selbstverlag on- und offline erscheint. Für diese ersten Überlegungen wurden zwei Beispiele aus dem Feld der prominenten Texte ausgewählt, ein Text von Eckhart Tolle und einer von Suzanne Segal, die gleich noch näher vorgestellt werden.

Zunächst wurde ein Facharzt für Psychiatrie/Psychotherapie gefunden, der einen solchen Dialog über dieses Thema grundsätzlich für sinnvoll hielt. Danach wurden die Texte Jetzt! Die Kraft der Gegenwart. Ein Leitfaden zum spirituellen Erwachen von Eckhart Tolle (2011) und Kollision mit der Unendlichkeit. Ein Leben jenseits des persönlichen Selbst von Suzanne Segal (2010) als Textgrundlage für das Gespräch vereinbart. Es folgte eine mehrwöchige Zeit für das Einzelstudium der Texte und danach wurden zwei Gespräche vereinbart, bei denen die Texte anhand von vorliegenden Auszügen gemeinsam interpretiert wurden. Diese gemeinsamen Interpretationen wurden aufgezeichnet und als Interview (nicht als Sprachdatum) verschriftet. ${ }^{4}$ Beide erhielten danach die Möglichkeit, die eigenen verschrifteten

4 Dies bedeutet z. B. auch, dass die intensive gesprächsbegleitende Rückmeldeaktivität des Interviewers in Form von Rückmeldepartikeln nicht (durchgängig) als eigener Gesprächsbeitrag bzw. in Partiturschreibweise dargestellt wird. 
Gesprächsbeiträge nach eigenem Ermessen zu ändern. Dieser Text letzter Hand geht in die folgenden Überlegungen ein. ${ }^{5}$ Das hier skizzierte Vorgehen hat sich als relativ aufwändig erwiesen, insbesondere die zeitliche Koordinierung, die Vorbereitungen, der relativ große Textumfang, das Erklären des Settings etc. machen diese Methode zu einem sehr langsamen Verfahren.

Um die Ergebnisse dieser beiden Gespräche soll es nun gehen, um zum einen das dialogische Interpretationsformat zu diskutieren, und um zum anderen die Frage zu klären, welche Erkenntnis wir über den Begriff des Normalen bzw. der Normalität daraus gewinnen können.

Methodisch lässt sich dieses Verfahren in den qualitativen Kanon einordnen, nämlich als neue Form des Experteninterviews, das „Dialogisches Experteninterview“ genannt werden soll: Nicht ein ${ }^{\star}$ e neutrale ${ }^{\star}$ r Fragende`r fragt, sondern zwei unterschiedliche Expert ${ }^{\star}$ innen lesen diskursiv den Text, wobei ein ${ }^{\star}$ e Expert ${ }^{\star}$ in damit eine (offengelegte) Fragestellung verfolgt. Es geht also nicht um eine Deutungsmusteranalyse des Interviewpartners wie im ,diskursiven Interview“ (Ullrich 2020), sondern um die dialogisch-hermeneutische Entfaltung eines gemeinsamen Textverständnisses bis hin zu elaborierten schriftlichen Artikulationen. Dieses kann und wird in der Regel auch reflexive und selbstreflexive Momente und damit auch Hinweise auf Deutungsmuster enthalten. Die Thematisierung von Deutungsmustern geschieht bei diesem dialogischen Format allerdings nicht nur beim Interviewten, sondern auch bei der Person, die das Interview führt. Beide sind insofern Peers, als sie Expert*innen auf ihrem jeweiligen Gebiet sind. Man könnte dieses Verfahren daher auch interdisziplinäre oder multiperspektivische Lektüre nennen, bei der eine Person die Fragestellung bestimmt.

Im Folgenden sollen nun die Textgrundlagen und die Lektüreergebnisse des Dialogischen Experteninterviews vorgestellt werden. Im ersten Interview ging es um den Text von Eckhart Tolle (2011), im zweiten Interview um den Text von Suzanne Segal (2010).

\section{Eckhart Tolles Jetzt!: Ewige Glückseligkeit oder wahnhaftes Erleben?}

Der Deutsch-Kanadier Eckhart Tolle wurde 1948 als Ulrich Leonard Tolle geboren und ist derzeit die bekannteste Person in der Szene der informellen Religiosität.

5 Aufgrund des Umfangs sind die vollständigen Interviews nicht für die vorliegende Publikation, sondern für einen größeren Projektrahmen vorgesehen. 
Seine Bücher haben eine Auflage von über 8 Millionen Exemplaren und werden in mehr als 30 Sprachen übersetzt. Er ist weltweit tätig und betreut Tolle-TV, eine Webinar-Plattform mit über 30 Millionen Teilnehmern. Sein erstes und nach wie vor bedeutendstes Buch ist das vorhin genannte Jetzt! Die Kraft der Gegenwart. Ein Leitfaden zum spirituellen Erwachen, das auch Textgrundlage für das dialogische Experteninterview war. ${ }^{6}$

Linguistisch ließe sich hier viel zur Textsorte, zur Textfunktion, zur Textstruktur und zum Stil der Texte Tolles sagen (vgl. dazu Liebert i. E.). Dies soll aber zu Gunsten der Ergebnisdarstellung der interdisziplinären Lektüre zurückgestellt werden. Im Folgenden sind: $\mathrm{P}=$ Dr. Markus Doetsch (Psychiater) und L = Prof. Dr. Wolf-Andreas Liebert (Linguist).

P: Eckhart Tolle kann anschaulich beschreiben, was er erlebt hat. Also die innere Wende, wenn man das so sagen darf. Dabei erkennt man ein häufig beschriebenes Muster bedeutsamer religiöser Erfahrung, die mir zum Beispiel aus vielen Textstellen des Alten und Neuen Testamentes und Bekehrungs- und Erweckungserlebnissen von Glaubensgründern bekannt ist. Nach einer ersten Phase tiefer existentieller Verunsicherung, Zerknirschtheit, oft auch Verzweiflung folgt eine alles verändernde, außergewöhnliche Erfahrung, die erstmals in das Leben der Person einbricht. Fast immer wird betont, dass dieses Erleben nicht selbst erzeugt wird, sondern einem irgendwie widerfährt oder sich eine wichtige Erkenntnis mit völliger Evidenz ergibt. Und danach ist das Leben nicht mehr wie zuvor. Das gilt als ein Hauptkriterium für eine bedeutsame religiöse oder allgemeiner spirituelle Erfahrung: das Angefasst-Werden oder Ergriffensein von etwas Anderem und danach bin ich nicht mehr derselbe und ich kann/möchte auch nicht mehr mein bisheriges Leben unverändert weiterführen. Eckhart Tolles Beschreibungen sind eigentlich noch im normalpsychologischen Bereich für kulturell vorbekanntes spirituelles Erleben, es sei denn, man zweifelt aus ideologisch-weltanschaulichen Gründen die Normalität von Religion und Spiritualität prinzipiell an. Und bezeichnend dafür, dass Eckhart Tolle eigentlich keine gravierende, also beispielsweise psychotische Erkrankung haben kann, ist seine fortbestehende Selbstreflexion in unmittelbarer Nähe zur Erfahrung und der Sachverhalt, dass er danach im Leben gut klarkommt, aus seinen Einsichten ein diskutierbares theoretisches System entwickelt und zwischenmenschlich scheinbar unbeeinträchtigt kontaktfähig bleibt. Einschränkend ist $\mathrm{zu}$ betonen, dass psychopathologische Aussagen zu Textdokumenten ohne persönlichen Kontakt zur erlebenden Person eigentlich nicht erfolgen sollten oder Einschätzungen zumindest mit größter Vorsicht und unter Vorbehalt zu treffen sind. Eckart Tolle beschreibt eine längere Phase der existentiellen Verzweiflung und des Unglücklichseins, dabei scheint er sich mit Fragen seiner Identität und des Lebenssinns beschäftigt zu haben. Ob es sich um eine klinisch relevante Depression handelte, lässt sich rückblickend nicht sicher entscheiden, aber auch nicht ausschließen. Unübersehbar kommt es dann zu einem Moment der völligen Klarheit und Einsicht, also eine Art Eingebung oder Erleuchtung. Ich gehe davon

6 Diese Textgrundlage (insb. S. 15-18) kann hier aufgrund der Umfangbegrenzung nicht wiedergegeben werden, ist aber als Buchpublikation gut verfügbar. 
aus, dass er zum besagten Thema lange gegrübelt und einiges gelesen hat. Der Kernsatz „ich kann mit mir nicht mehr leben“ mit der sich anschließenden Ausführung „Bin ich einer oder zwei? Wenn ich nicht mit mir selbst leben kann, muss es zwei von mir geben, das Ich und das Selbst, mit denen ich nicht leben kann“ verweist auf eine vorausgehende Auseinandersetzung mit diesen psychologischen und philosophischen Begriffen sowie vermutlich auch mit in weitestem Sinne buddhistischer Literatur. Zwar existieren bedeutsame Erweckungsund Bekehrungsbeschreibungen, in denen die Person ohne jegliche Vorentwicklung von einer göttlichen Erfahrung oder Erleuchtung getroffen wird (und die auch gerne als Beweis für eine wirkmächtige Transzendenz angeführt werden), dennoch sind spirituelle Erfahrungen häufig durch Suchen und Sehnsüchte vorgeformt, wie meines Erachtens auch bei Eckhart Tolle. Anschließend berichtet er von einem „Energiewirbel“, einem Hineingesaugtwerden in eine Leere und er hört die Worte „Wehre dich nicht“. Hier verlässt er den Bereich des uns vertrauten Alltagsbewusstseins und macht Erfahrungen, die nicht jedem Menschen geläufig sind oder am ehesten in ähnlicher Form sich in einem Traum ereignen können, wobei er definitiv in diesem Moment nicht träumt. Psychopathologisch könnte man die Frage stellen, ob er möglicherweise Halluzinationen erlebte und seine Erfahrung ein wahnhaftes religiöses Erleben darstellte. Dagegen spricht, dass die Stimme aus seiner Brust kam und nur kurzzeitig vorlag, am ehesten handelt es sich um komplexe imaginative Erfahrungen, die durchaus eine sehr körperlich konkrete Dimension annehmen können, ohne dass sein Realitätsgefühl bleibend beeinträchtigt wird. Anschließend berichtet er von einem ausgesprochenen Glückszustand, ein Hochgefühl mit einer Art innerer Erleuchtung. Dabei bleibt er sehr geordnet, eine Kontinuität des Erlebens mit Sinnhaftigkeit ist gegeben, meines Erachtens ist dies nicht psychotisch.

L: Aber in der Zeit ist er ja schon aus dem Alltag rausgefallen. Er sitzt auf der Parkbank...

P: Zugegebenermaßen ist diese Passage rückblickend besonders schwierig einzuschätzen. 5 Monate in einem ununterbrochenen Gefühl der Glückseligkeit sind selten und es wäre sehr hilfreich, ihn damals gesprochen und beobachtet zu haben. Psychiatrisch könnten theoretisch eine hypomanische Phase, eine Glückspsychose oder allgemeiner ein positiver religiöser Wahn vorliegen. Selten halten diese Zustände aber kontinuierlich an, oft zeigen sich dabei erhebliche Schwankungen und gerade religiöse Wahnerlebnisse kippen durchaus oft in negative Wahneinfälle, Rettung und Untergang wechseln sich ab mit durchaus auch erheblichen Gefahren für die betroffene Person. Wichtig ist die Differenzierung, ob er nur aus dem Alltag fällt oder auch aus der Realität. In Anbetracht seines weiteren Lebensverlaufes und einer doch eindrucksmäßig fortbestehenden basalen Lebens- und Steuerungsfähigkeit auch während seiner Glücksphase scheint mir auch rückblickend keine schwerwiegende psychiatrische Erkrankung im Sinne einer Psychose vorgelegen zu haben. Mit Abstand betrachtet handelt es sich um einen besonderen, von der üblichen westlichen Norm abweichenden Lebenslauf, der nun aber doch als „besonders erfolgreich“ einzustufen ist und das mit erstaunlicher Kontinuität. Hierzu könnte man natürlich psychodynamische oder allgemeiner entwicklungspsychologische Vermutungen anstellen, die im Zusammenhang mit Bekehrungs- und Erleuchtungserlebnissen vermutet werden, die aber ohne eine therapeutische Beziehung auch etwas Anmaßendes haben und nur eine Sichtweise auf eine primär existentielle Erfahrung darstellen.

(Interview 1, 29.1.2019) 
Diese Passage zeigt den hohen Reflexionsgrad des Psychiaters, der sich ständig vergegenwärtigt, dass es sich hier um eine Schrifttext-Interpretation handelt und er daher Fragen simuliert, die in einer Face-to-Face-Situation gestellt werden müssten. Das vorsichtige Abtasten von möglichen Krankheitsbildern zeigt aber auch eine Gradwanderung zwischen religiösem Erleben und einer möglichen Krankheit. Dabei werden auch mögliche Vorurteile atheistischer oder religiöser Psychiater genannt und die genannten Klassifikationssysteme abgetastet.

Um die Grenzsituation stärker zu elizitieren, verschärfte der Interviewer L. daher die Situation, indem er den Erzähler der Tolle'schen Erwachenserzählung als Störfall in eine psychiatrische Gesprächssituation versetzte:

L: Wenn wir nun annehmen, dass Herr Tolle oder eine Person mit gleicher biografischer Erzählung in die Praxis eines Psychiaters gekommen wäre. Würde der Psychiater dann eine der eben genannten Diagnosen fällen?

P: Er würde sicherlich versuchen, das Erleben der Person möglichst exakt zu erfassen und vertiefende Fragen stellen, um zwischen einer vielleicht auch den Psychiater irritierenden außergewöhnlichen Erfahrung und einer akuten Erkrankung zu unterscheiden. Wichtige Fragen wären, ob die Person noch einen Perspektivwechsel übernehmen kann, ob er zwischen inneren Einsichten und äußerer Alltagswelt unterscheiden kann, ob er sein Erlebnis als von außen gemacht erfährt und wie die Stimmung und sein Antrieb einzustufen sind, nicht fehlen darf die Frage nach suizidalen oder aggressiven Impulsen. Ergeben sich dabei erhebliche Auffälligkeiten, könnte eine der oben erwähnten Diagnosen zustande kommen, ansonsten würde man sich für ein abwartendes Verhalten entscheiden.

L: Ich meine auch speziell in der Phase, in der er diesen Schub hatte. Wenn er, statt sich fünf Monate glückselig auf die Parkbank zu setzen, in die Ambulanz gekommen wäre. Nach dem Erstgespräch hätte der Psychiater dann sowas gesagt wie ,Das war eine einmalige Episode in seinem Leben.' oder ,Ein wunderbarer spiritueller Durchbruch!' Wäre das möglich gewesen?

P: Das bleibt eine rückblickend schwierige Frage. Festzuhalten ist nochmals, dass der Psychiater die Aufgabe hat, eine mögliche Erkrankung oder Gefährdung zu erkennen und eventuell Hilfe anzubieten. Wichtig ist festzuhalten, dass Herr Tolle definitiv nicht in eine Ambulanz oder Praxis kam. Daraus lässt sich schließen, dass er damals keinen Leidensdruck hatte und auch sein durchaus vorhandenes Umfeld keine Veranlassung für eine Untersuchung sah, was für ein doch zumindest einigermaßen realitätsangepasstes Verhalten spricht. Natürlich ist es denkbar, dass je nach Interaktion und Rückmeldungen von Herrn Tolle eine psychiatrische Diagnose gestellt worden wäre und man eine Verlaufsbeobachtung angeboten hätte, um einen eventuell doch eintretenden Stimmungswechsel und Gefährdungsmomente zu erkennen. Gratulieren zum „spirituellen Durchbruch“ könnte man nur, wenn man sich zutraut, in dem jeweiligen spirituellen Kontext der Person kundig zu sein und es auch tatsächlich als authentische Erfahrung einschätzt. Psychiater sind prognostisch meistens vorsichtig, da man 
im klinischen Alltag schon viele Überraschungen erlebt hat und die Kehrseite von erkrankungsbedingten Glücksgefühlen zu Genüge kennengelernt hat.

L: „Kehrseite vom Glücksgefühl“ heißt dann...?

P: Die gehobene oder ekstatische Stimmung kippt in einen depressiven Zustand oder in ein negatives Wahnerleben wie zum Beispiel der Verdammnis, Schuldigkeit oder Nichtigkeit. Inwiefern es bei „echten spirituellen Erfahrungen“ zu Krisen kommen kann, ist schwierig einzuschätzen, meines Wissens hatten aber auch beglückte und erleuchtete Personen zumindest im christlichen Kontext schlimme Phasen des Zweifels und der „dunklen Nacht“. Psychiatrische Erkrankungen können mit lange gehobener Stimmungslage einhergehen, häufiger sind aber polare Umschwünge wie der Wechsel zwischen Manie und Depression, extreme Angst und Glück in der Angst-Glückspsychose und Auserwählung und Verdammnis im religiösen Wahn. Damit verbunden sein können Suizidalität und selten auch Fremdgefährdung aufgrund der Realitätsverkennung.

(Interview 1, 29.1.2019)

Solange sich also niemand an einem Menschen stört, der über einen längeren Zeitraum nichts anderes zu brauchen scheint, als auf einer Bank zu sitzen, und dabei auch noch permanent ein Glücksgefühl ausstrahlt, wird es auch keine Interaktion mit der Psychiatrie geben. Allerdings kann ,je nach Interaktion und Rückmeldungen“ dann doch noch „eine psychiatrische Diagnose gestellt“ werden. Auch Eckhart Tolle hätte daher unter bestimmten Umständen ein ,Fall“ werden können. Der Grund liegt aber nicht in einer wie auch immer gearteten ,Böswilligkeit‘ des Psychiaters, sondern in den gesellschaftlichen Mechanismen, die einsetzen, wie der Frage nach der Haftung, die eintritt, wenn sich der Psychiater geirrt haben sollte, in seinen Erfahrungen, wie ekstatische Zustände „kippen“ können, wie wenig Vertrauen er letztlich in die Stabilität eines solchen Glücksgefühls setzen kann, in seiner Unsicherheit, religiöse Zustände erkennen und einschätzen zu können.

\section{Suzanne Segals Kollision: Erwachen in den Alptraum?}

Die Textgrundlage des zweiten Gesprächs stammt aus Suzanne Segals Autobiografie Kollision mit der Unendlichkeit. Ein Leben jenseits des persönlichen Selbst (2010). Es handelt sich hier ebenfalls um ein weit verbreitetes Buch, das auch viele Reaktionen und Kommentare in Publikationen und im Internet hervorgerufen hat. Suzanne Segal war mehrfach vergeblich in psychiatrischer Behandlung 
und hat sich mit verschiedenen spirituellen Lehrern ausgetauscht, bevor sie selbst spirituelle Lehrerin wurde. Sie verstarb früh an einem Hirntumor. ${ }^{7}$

Der Fall Suzanne Segal ist hinsichtlich der Frage, ob es sich um ein spirituelles Erwachen oder um eine Form psychischer Krankheit handelt, stark umstritten. Sie selbst thematisiert dies in ihrem Buch immer wieder. Ihr Pendeln zwischen spirituellen Lehrern und Psychiatern ist ebenfalls Ausdruck dieser Ambivalenz. Dieser Diskurs soll an dieser Stelle allerdings nicht weiter ausgeführt werden.

Anders als im ersten Text (Tolle 2011) erzählt Suzanne Segal (2010) viele Details über ihr Alltagsleben und ihre Familiengeschichte, so dass die Interpretation des Psychiaters viele Anknüpfungspunkte findet und das Interview über Segals Erwachenserzählung deutlich anders verläuft als das erste.

P: Beim Lesen des Segaltextes entsteht schnell der Eindruck, dass es sich auf der Phänomenebene um ein Depersonalisationssyndrom handelt. Darunter versteht man ein umschriebenes Erkrankungsbild, das unter dem zusammenhängenden Begriff Depersonalisations- und Derealisationssyndrom mit einer eigenständigen ICD- und auch DSM-Nummer versehen ist. Viele Diagnosekriterien scheinen zuzutreffen, wobei es individuell ausgestaltet ist und einige Angaben vorliegen, die schon etwas atypisch oder exzentrisch sind und die Frage aufwerfen, ob die Symptomatik darüber hinaus geht und zusätzliche Diagnosen zu stellen sind wie eine eng damit verbundene dissoziative Störung und ein depressives Syndrom. In der Sekundärliteratur zu Suzanne Segal im Internet, beispielsweise bei Wikipedia, wird erwähnt, dass sie psychiatrisch vorgestellt wurde und mehrere dieser Diagnosen vermutet wurden. (....) Depersonalisation beschreibt einen Zustand, in dem man sich selbst nicht richtig spüren kann, der Zugang zur eigenen Person und dem emotionalen Erleben ist beeinträchtigt oder fehlt fast völlig, man kommt sich fremd vor und kaum trennbar davon erscheint einem die Umgebung unwirklich und fern oder fremd. Es handelt sich nicht um ein psychotisches Erleben, wobei es auch bei psychotischen Erkrankungen zu Depersonalisationen kommen kann, die dann aber eine nochmals extremere Dimension annehmen und mit dem Gefühl des Gemachten, also durch eine Außeneinwirkung verursacht, verbunden sind. Das stellt immer ein Hauptkriterium für psychotisches Erleben dar, der Eindruck oder die Überzeugung der äußeren Beeinflussung. Beim Depersonalisations-Derealisationssyndrom habe ich nicht mehr den vollen Zugriff auf meine Person und die Welt. Aber ich habe noch eine realistische Wahrnehmung, dass ich dies gerade erlebe. Man könnte auch sagen, „mir kommt alles unwirklich vor oder ich bin nicht richtig bei mir“. Im normalen Sprachgebrauch existieren einige gängige Formulierungen zu dieser Erlebnisform. Dass sie selbst ihr Erleben als „Leerwerden“ deutet, basiert vermutlich auf ihrer Vorbildung. Sie hat sich im Vorfeld mit vielen spirituellen Fragen beschäftigt. Unter anderem war sie schon bei Gurus gewesen und hat sich mit indisch-buddhistischen Schriften auseinandergesetzt.

(Interview 2, 12.3.2019)

7 Wie eben bei der Analyse von Eckhart Tolles Jetzt! (2011) kann der Text von Suzanne Segal (insb. S. 64-70) hier nicht dargestellt werden; auch dieser Text ist jedoch gut verfügbar. 
In diesem Fall legt sich der Psychiater wiederum in Würdigung möglicher ekstatischer Zustände eindeutig auf eine Diagnose fest:

P: Also die meisten Psychiater würden in Kenntnis der Diagnosekriterien und des Erkrankungsbildes ruhig reagieren und beispielsweise sagen: „Ja, wissen Sie, was sie da erleben, ist gar nicht so ein seltenes Phänomen, das aus vielfältigen Gründen auftreten kann. Sie müssen nicht befürchten, dass sie verrückt werden und Sie dürfen auch darauf bauen, dass es wieder besser wird“. So könnte eine erste Antwort lauten.

(Interview 2, 12.3.2019)

Der Text von Suzanne Segal erzählt ihre Geschichte im Gegensatz zu Tolles Text bis ins kleinste Detail. Detailreichtum und Detaillierungsgrad sind ein wesentliches Stilmittel ihrer Erzählung. Diese Erwachenserzählung wurde vom Psychiater im Verlauf durchgängig als sogenanntes Derealisations-/Depersonalisationssyndrom interpretiert. Durch Segals Darstellung ihrer Familienkonstellationen wurde im Gesprächsverlauf auch die Familiensituation in die Deutung einbezogen, was hier nicht im Einzelnen dargestellt werden kann. Dass Suzanne Segals Autobiografie das Dokument eines spirituellen Erwachens ist, wurde vom Psychiater jedoch klar bestritten, dagegen wurde eine psychische Erkrankung angenommen. Segals teils ekstatische, hyperbolische Beschreibungen, insbesondere die Darstellung der sie behandelnden Psychiater und Psychotherapeuten, die ihren Fall in kein Schema einordnen konnten, wurden als „histrionisch“ klassifiziert:

P: Die beschriebenen Erfahrungen kann ich schwerlich als spirituelles Erlebnis deuten. Ich finde es im gesamten Text sehr irritierend, dass sie eine durchweg negative Erfahrung als spirituelles Erleben einstuft. Es ist kein glücklicher oder erlöster Zustand, es scheint eine einzige Quälerei zu sein, so wie sie sich beschreibt. Eine lebensdienliche Erkenntnis scheint zu fehlen. Völlig irritierend ist auch die Erklärung eines von ihr aufgesuchten spirituellen Lehrers, der sie zu ihren schmerzhaften und beängstigenden Erfahrungen beglückwünscht. Man fragt sich, ob der Lehrer überhaupt ernsthaft zugehört hat oder zynisch veranlagt ist.

(Interview 2, 12.3.2019)

Die Erzählerin wird in der gemeinsamen Lektüre als eine Person in Not gesehen, die innerhalb ihrer Erzählung jedoch als unheilbar erscheint und sich als erwacht bezeichnet. Dieses ,Erwachen‘ wird vom Psychiater als psychische Erkrankung gedeutet, die prinzipiell heilbar gewesen wäre. Es gibt jedoch einige Textstellen in Segals Text, die den Psychiater dennoch leicht unsicher werden lassen, so dass dieses zweite dialogische Experteninterview zwar zu einem klareren Ergebnis als das erste kommt, aber bestimmte Unsicherheiten eben bestehen bleiben. 


\section{Fazit}

Die linguistische Hypothese über Ähnlichkeiten religiöser Sprache mit der Sprache von psychisch Kranken hat durch die beiden Interviews zu grundlegenden Fragen über die Grenzen von Normalität und die Einbettung dieser Frage und ihrer möglichen Antworten in vorgängige Normalitätsdiskurse geführt. Einerseits zeigt sich die Macht des ICD und des DSM in der psychiatrischen Deutung. Die Rolle von ICD und DSM ist selbst Gegenstand psychiatrischer Diskurse, sogar für die hier diskutierte Frage nach der Normalitätsgrenze religiöser Erfahrung und psychischer Erkrankung (vgl. z.B. Allmon 2013). Auch der interviewte Psychiater zeigte sich in den Interviews dieser Diskursmacht bewusst, es finden sich häufig reflektierende und auch selbstironische Passagen dazu. Dies unterstreicht die mächtige Diskursposition, die ICD und DSM einnehmen. Diskurslinguistisch müssen sie daher als zentrale Deutungsmuster betrachtet werde, die in jedem psychiatrischen Gespräch zum Tragen kommen. Sie besitzen im Sinne Foucaults Diskursmacht und können nach Bruno Latours Akteur-Netzwerk-Idee als eigene Akteure betrachtet werden, auch wenn wechselnde Autorenkollektive dafür verantwortlich zeichnen (Latour 2014).

Ein weiteres Ergebnis ist die Positionierung in Bezug auf Transzendenz: Der Psychiater vertrat die Ansicht, dass ein Psychiater, je nachdem, wie er sich zur Transzendenz positioniert, religiöse Erlebnisse anders interpretieren wird. Dies stellt eine anregende Hypothese für weitere Untersuchungen dar.

Das agonale Zentrum zwischen transzendenter und non-transzendenter Positionierung wurde kurz historisch skizziert, in den Interviews wird es auch mehrmals thematisiert. Die Kategorie der trans-transzendenten Positionierung könnte helfen, das Feld in grundsätzlicher Weise offener zu gestalten. Neben der Frage nach geschlossener Gewissheit in den transzendenten und non-transzendenten Positionierungen könnte so auch die Frage nach dialogischem Glauben, also einer offenen Gewissheit, gestellt werden. Diese müsste aber von vornherein insbesondere durch eine geeignete Textauswahl eingeleitet werden.

Es wurde in den Interviews auch deutlich, dass es in bestimmten Kontexten durchaus möglich wäre, dass selbst reflektierte Psychiater bestimmte religiöse Erfahrungen als psychische Krankheit deuten würden, ja sogar müssten, selbst wenn für sie darin eine große Unsicherheit liegen würde. Andererseits gibt es klare Fälle mit geringer Unsicherheit, wie die rätselhafte Erwachenserzählung von Suzanne Segal. Hier erweist sich das klinische Deutungsmuster des Derealisations-/Depersonalisationssyndroms als durchgängiger Interpretationsleitfaden, der sich durch die gesamte Segal'sche Erzählung zieht. 
Die Methode des Dialogischen Experteninterviews konnte im Verlauf des Interviews immer deutlicher ausgearbeitet werden. Ausgangspunkt war die Annahme, etwas (teilnehmend) beobachten zu können und ,Daten' zu erheben. Diese Annahme entpuppte sich zunehmend als illusionär. Durch den Interviewprozess wurde die Datenerhebung immer mehr zu einer Ko-Kreation: Die Vorstellung von zu gewinnenden Daten wurde schließlich ganz verworfen zugunsten eines gemeinsam zu schreibenden (Teil-)Textes über die Phänomene, über die gesprochen wurde. Aus dem ursprünglich einmal geplanten ,Datum‘ trat immer deutlicher ein Gesprächspartner mit eigenen Vorstellungen, eigenem Stil, eigenem Wissen und einer eigenen Sprache hervor, der in seiner Eigenständigkeit und Widerständigkeit nun in diesem Text Autorschaft beanspruchen konnte. Es handelt sich also um eine Ko-Autorschaft an den dialogischen Teiltexten innerhalb des Aufsatzes. ${ }^{8}$

\section{Offene Fragen}

Die ersten Annäherungen an einen Antworthorizont zur Frage, wie die Grenze zwischen psychischer Erkrankung und religiöser Erfahrung zu ziehen sei und welche Rolle sprachliche und Erfahrungsmerkmale aus religionslinguistischer und psychiatrischer Sicht spielen können, werfen unmittelbar neue Fragen auf, noch bevor die zuvor gestellten in befriedigender Weise beantwortet wären: Wie mächtig sind die Werke ICD und DSM wirklich? Ist die Verführung ihrer Verständigungseinheit so groß, dass sie die Frage nach ihrer Validität vergessen macht? Können religiöse Erfahrungen überhaupt in einer auf Störung angelegten Klassifikation als solche erkannt und damit von einer weiteren Diagnosestellung ausgeschlossen werden? Gibt es religiöse Menschen, die aufgrund der eben genannten Faktoren falsch diagnostiziert wurden? Welche Erwachenserzählungen sind letztlich Berichte von psychischer Not und Krankheit? Hierzu gibt es bereits grundlegende Diskurse und auch sprachreflexive Arbeiten in der Psychiatrie (z.B. Peteet et al. 2011; Chandler 2012), die sich eine Diskurslinguistik aneignen müsste, um in ein ernsthaftes Gespräch über diese Frage eintreten zu können.

Neben vielen weiteren Fragen im Zuge der Interviews - ob es etwa mit dem Bedeutungsverlust der christlichen Kirchen auch einen Wandel in der religiösen Psychopathologie gebe - stellt sich schließlich die Frage nach der Wirksamkeit der Alternativen psychiatrischer Deutungen von Wahnsinn, wie z.B. solchen aus

8 Für die vorliegenden Interviews wurde der Gesprächspartner gefragt, in welcher Weise er genannt werden will. 
dem künstlerischen Bereich. Dies regt insbesondere zur weiteren Frage an, ob sich in der spirituellen Literatur informeller Religiosität nicht selbst auch Deutungen von Wahn finden. Ein erster Blick darauf ist vielversprechend, müsste aber noch weiter untersucht wurden.

\section{Literatur}

Allmon, Allison L. (2013): Religion and the DSM: From Pathology to Possibilities. Journal of Religion and Health 52 (2), 538-549.

American Psychiatric Association (APA) (Hrsg.) (2018): Diagnostisches und Statistisches Manual Psychischer Störungen. DSM-5. 2., korrigierte Aufl. Göttingen, Bern, Wien u.a.: Hogrefe.

Bochinger, Christoph, Martin Engelbrecht, Winfried Gebhardt (Hrsg.) (2009): Die unsichtbare Religion in der sichtbaren Religion. Formen spiritueller Orientierung in der religiösen Gegenwartskultur. Stuttgart: Kohlhammer.

Bucher, Anton A. (2007): Psychologie der Spiritualität. Handbuch. Weinheim, Basel: Beltz. Chandler, Emily (2012): Religious and Spiritual Issues in DSM-5: Matters of the Mind and Searching of the Soul. In: Issues in Mental Health Nursing, 33(9), 577-582.

Dilling, Horst, Werner Mombour, Martin H. Schmidt (Hrsg.) (2015): Internationale Klassifikation psychischer Störungen. ICD-10. Göttingen, Bern, Wien u.a.: Hogrefe.

Felder, Ekkehard (Hrsg.) (2006): Semantische Kämpfe. Macht und Sprache in den Wissenschaften. Berlin, New York: De Gruyter.

Finzen, Asmus (2018): Normalität. Die ungezähmte Kategorie in Psychiatrie und Gesellschaft. Köln: Psychiatrie-Verlag.

Foucault, Michel (2018): Wahnsinn und Gesellschaft. Eine Geschichte des Wahns im Zeitalter der Vernunft. 23. Aufl. Frankfurt a.M.: Suhrkamp.

Freud, Sigmund (1927): Die Zukunft einer Illusion. Gesammelte Werke. Bd. 14, 325-380. https://www.textlog.de/sigmund-freud-zukunft-illusion.html (letzter Zugriff: 29.09.2018).

Gebhardt, Winfried, Martin Engelbrecht, Christoph Bochinger (2005): Die Selbstermächtigung des religiösen Subjekts. Der „spirituelle Wanderer“ als Idealtypus spätmoderner Religiosität. Zeitschrift für Religionswissenschaft 13, 133-151.

Grom, Bernhard (2007): Religionspsychologie. 3., vollst. überarb. Aufl. München: Kösel. Habermas, Jürgen (2012): Nachmetaphysisches Denken II. Aufsätze und Repliken. Berlin: Suhrkamp.

James, William (1997): Die Vielfalt religiöser Erfahrung. Eine Studie über die menschliche Natur (Insel Taschenbuch 1784). Frankfurt a.M.: Insel.

Knoblauch, Hubert (2009): Populäre Religion. Auf dem Weg in eine spirituelle Gesellschaft. Frankfurt a.M.: Campus.

Lasch, Alexander (2005): Beschreibungen des Lebens in der Zeit. Zur Kommunikation biographischer Texte in den pietistischen Gemeinschaften der Herrnhuter Brüdergemeine und der Dresdner Diakonissenschwesternschaft im 19. Jahrhundert. Münster u.a.: LIT. Latour, Bruno (2014): Existenzweisen. Eine Anthropologie der Modernen. Berlin: Suhrkamp. 
Liebert, Wolf-Andreas (2017): Religionslinguistik. Theoretische und methodische Grundlagen. In Alexander Lasch \& Wolf-Andreas Liebert (Hrsg.), Sprache und Religion. (Handbücher Sprachwissen 18), 7-36. Berlin, Boston: De Gruyter.

Liebert, Wolf-Andreas (2018): Können wir mit Engeln sprechen? Über die eigenartige (Un-) Wirklichkeit der Verständigung im Religiösen. In Ekkehard Felder \& Andreas Gardt (Hrsg.), Wirklichkeit oder Konstruktion? Sprachtheoretische und interdisziplinäre Aspekte einer brisanten Alternative, 162-193. Berlin, Boston: De Gruyter.

Liebert, Wolf-Andreas (i. E.): Lost in Enlightenment. Zur sprachlichen Darstellung von Erwachenserlebnissen in spätmoderner, informeller Religiosität. In Moritz von Kalckreuth (Hrsg.), Philosophische Anthropologie und Religion. Religiöse Erfahrung, soziokulturelle Praxis und die Frage nach dem Menschen (Philosophische Anthropologie). Berlin, Boston: De Gruyter.

Lukoff, David (2017): Die Kategorie „Religiöses oder Spirituelles Problem“ im DSM-IV und DSM-5. In Liane Hofmann \& Patrizia Heise (Hrsg.), Spiritualität und spirituelle Krisen. Handbuch zu Theorie, Forschung und Praxis, 32-46. Stuttgart: Schattauer.

Marx, Karl (1956): Zur Kritik der Hegelschen Rechtsphilosophie. Einleitung. In Karl Marx \& Friedrich Engels, Werke. Band 1. Berlin. http://www.zeno.org/nid/2000921464X (letzter Zugriff: 29.09.2018).

Mönter, Norbert (Hrsg.) (2007): Seelische Erkrankung, Religion und Sinndeutung. Bonn: Psychiatrie-Verlag.

Mundhenk, Ronald (2010): Lebt Gott in der Psychiatrie? Erkundungen und Begegnungen. Neumünster: Paranus.

Peteet, John R., Francis G. Lu, William E. Narrow (Hrsg.) (2011): Religious and Spiritual Issues in Psychiatric Diagnosis: A Research Agenda for DSM-V. Arlington: American Psychiatric Association.

Reiser, Franz (2018): Menschen mehr gerecht werden: Zur Religiosität bzw. Spiritualität von Patientinnen und Patienten in Psychiatrie und Psychotherapie. Würzburg: Echter.

Schödlbauer, Michael (2016): Wahnbegegnungen. Zugänge zur Paranoia (Anthropologische Psychiatrie 1). Köln: Psychiatrie-Verlag.

Ullrich, Carsten G. (2020): Das Diskursive Interview. Methodische und methodologische Grundlagen. 2. Aufl. Wiesbaden: Verlag für Sozialwissenschaften.

Vaitl, Dieter (2012): Veränderte Bewusstseinszustände. Grundlagen - Techniken Phänomenologie. Stuttgart: Schattauer.

Viveiros de Castro, Eduardo (2016): „Perspektiventausch“. In Irene Albers \& Anselm Franke (Hrsg.), Animismus. Revisionen der Moderne, 73-93. Zürich: Diaphanes.

\section{Quellen}

Segal, Suzanne (2010): Kollision mit der Unendlichkeit. Ein Leben jenseits des persönlichen Selbst. 4. Aufl. Reinbek bei Hamburg: Rowohlt.

Tolle, Eckhart (2011): Jetzt! Die Kraft der Gegenwart. Ein Leitfaden zum spirituellen Erwachen. 11. Aufl. Bielefeld: Kamphausen. 
